\title{
BEAM LOADING COMPENSATION FOR THE LOW FREQUENCY LINACS AND COMPRESSORS IN THE NLC *
}

\author{
Zenghai $\mathrm{Li}^{\dagger}$, T.O. Raubenheimer and Roger Miller \\ Stanford Linear Accelerator Center, Stanford University, Stanford, CA 94309
}

\begin{abstract}
The low frequency rf linacs and bunch compressors in the NLC are heavily loaded by beam currents up to 0.9 A. Beam loading generates bunch-to-bunch energy deviations, which need to be compensated to operate with long bunch trains in the linear collider. There are two basic compensation techniques, namely $\Delta T$ and $\Delta F$ schemes. The $\Delta F$ scheme is operationally straightforward but requires additional rf systems which are powered at slightly different frequencies. In contrast, the $\Delta T$ scheme does not require any additional $\mathrm{rf}$ and is thus expected to be less expensive. Here, the loading compensation is realized by early injection combined with rf phase and amplitude modulation. The phase/amplitude modulation is performed with fast phase shifters at low rf power before amplification by the klystrons. With combined phase and amplitude modulation, the $\Delta T$ scheme can be applied to both the accelerator and compressor linacs. In this paper, we present simulations of the $\Delta T$ compensation scheme for the NLC low rf accelerator and compressor linacs. We discuss different rf configurations using the $\Delta T$ scheme as well as some issues related to phase/amplitude modulations in such layouts.
\end{abstract}

\section{INTRODUCTION}

The Next Linear Collider (NLC) is an $e^{+} e^{-}$collider based on "high-frequency" X-band (11.4 GHz) rf power. The collider will be capable of achieving a luminosity in excess of $10^{34} \mathrm{~cm}^{-2} \mathrm{sec}^{-1}$ at a center-of-mass energy of $1 \mathrm{TeV}[1,2]$ and will have the capability to be expanded to $1.5 \mathrm{TeV}$.

The $e^{+}$and $e^{-}$injector complex for the NLC produces the low emittance bunch trains to be accelerated in the Xband linacs. The complex includes six L-band $(1.428 \mathrm{GHz})$ and four S-band $(2.856 \mathrm{GHz})$ linacs and compressors, listed in Table 1, which accelerate the beams to $10 \mathrm{GeV}$ and operate heavily beam-loaded. All these "low-frequency" linacs will be powered with klystrons using SLED-I rf pulse compression systems [3]. In a SLED-I pulse compression system, the SLED cavities are charged by a long klystron pulse. The stored energy is then extracted by reversing the klystron phase and, for typical parameters, power gains of roughly 3-4 can be attained.

A typical SLED-I output is shown in Fig. 1a. The power of the pulse has a maximum immediately after the klystron phase reversal and then decays exponentially. The acceleration voltage generated by such a pulse in a traveling wave

\footnotetext{
${ }^{*}$ Work supported by the DOE, contract DE-AC03-76SF00515.

† Email:lizh@slac.stanford.edu
}

structure is shown in Fig. 1b. The acceleration reaches maximum after a structure fill-time, and then decreases due to the drooping rf power input. The loaded voltage seen by the bunch train is the sum of the rf voltage and the beam loading voltage and is generally not constant along the bunch train; it depends on the structure, klystron, and beam parameters. The energy deviation along the train will have a direct impact on beam transport and luminosity of the collider and thus compensating the energy deviation is a critical requirement in the linear collider design.

Table 1: NLC Low rf frequency linacs

\begin{tabular}{|l|l|l|l|l|l|}
\hline Linac & Freq $^{*}$ & $\begin{array}{l}\mathrm{E} \\
(\mathrm{GeV})\end{array}$ & $\begin{array}{l}Q_{b} \\
\left(10^{10}\right)\end{array}$ & $\begin{array}{l}\mathrm{I} \\
(\mathrm{A})\end{array}$ & BLC \\
\hline$e^{+}$capture & $\mathrm{L}(1)$ & 0.250 & 7.9 & 4.5 & $\Delta F$ \\
\hline$e^{+}$booster & $\mathrm{L}(1)$ & 1.75 & 1.6 & 0.91 & $\Delta T$ \\
\hline$e^{+}$drive & $\mathrm{S}(1)$ & 6.0 & 1.45 & 0.83 & $\Delta T$ \\
\hline$e^{-}$booster & $\mathrm{S}(1)$ & 1.98 & 1.45 & 0.83 & $\Delta T$ \\
\hline$e^{-}, e^{+}$pre & $\mathrm{S}(2)$ & 8.0 & 1.15 & 0.66 & $\Delta T$ \\
\hline $\mathrm{EC}, \mathrm{BC} 1$ & $\mathrm{~L}(2 \times 2)$ & $\sim 0.1$ & 1.45 & 0.83 & $\Delta T$ \\
\hline
\end{tabular}
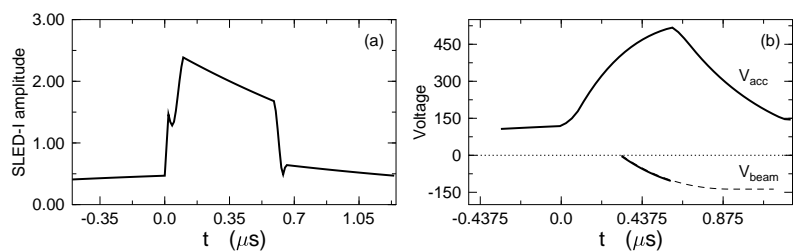

Figure 1: The pulse profile of a SLED-I system and the acceleration and beam voltages generated by a traveling wave structure. a) SLED-I amplitude; b) generator and beam induced voltages.

\section{BEAM LOADING COMPENSATION}

In the SLED-I driven systems, both the acceleration and beam loading voltages are transient. The goal of compensation is to obtain a uniform loaded voltage. In the following sections we will describe the $\Delta T$ and $\Delta F$ compensation schemes.

\section{$2.1 \Delta F$ scheme}

The beam voltage is compensated using additional rf structures operating at slightly different frequencies. The bunches in a bunch train are accelerated on different $\mathrm{rf}$ phases in these structures and the compensation that can be attained is proportional to the frequency difference $\Delta F$. However, to maintain a roughly linear compensation along 
the train, the frequency difference $\Delta F$ is limited by the bunch train length. For the NLC bunch train, $\Delta F$ should not be more than a couple of $\mathrm{MHz}$.

The advantage of the $\Delta F$ scheme is that it is quite flexible. The input power to the compensation structures can be scaled to compensate different beam loading conditions. However, the disadvantages of the $\Delta F$ scheme are the extra off-frequency rf systems required and the non-local correction since the compensation sections are distributed along the linac. Finally, the residual energy spread in this scheme is proportional to $\Delta F^{2}$, and it adds in phase throughout the linac regardless of the sign of $\Delta F$.

\section{$2.2 \Delta T$ scheme}

The $\Delta T$ compensation scheme uses the "natural" rising slope of the acceleration voltage as the structure fills to cancel the beam loading voltage. Complete cancellation requires, during the beam time,

$$
\frac{d V_{a c c}}{d t}=-\frac{d V_{b}}{d t}
$$

The profile of the acceleration voltage, Fig. 1, shows that the rising slope of the acceleration voltage decreases with time. This indicates that for a given beam loading current, there is an optimal fill-time for the structure such that condition Eq. (1) is satisfied. For the $\Delta T$ scheme to operate efficiently, the optimal fill-time of the structure should be much longer than the bunch train length. At very high beam currents, the efficiency of the $\Delta T$ scheme can be poor due to short optimal fill-times-in such cases, the $\Delta F$ scheme should be considered.

There are number of advantages of the $\Delta T$ scheme. First, the scheme does not require additional rf systems. Second, the compensation is local and the energy deviation is minimized at the end of every structure. Finally, the residual error of the compensation is uncorrelated and does not accumulate. However, the structure design for optimal compensation is tightly coupled to the beam and rf parameters. Once the structure is optimized for a given current, the compensation at other loading conditions will not be perfect. To compensate at different loading conditions, amplitude modulation of the SLED-I pulse is needed to reshape the acceleration profile. Similarly, in the compressor linacs where the beam loading voltage is $90^{\circ}$ out-of-phase with the acceleration voltage, phase modulation is needed to produce a component in phase with the loading voltage. Amplitude modulation is also required to obtain a flat compression voltage.

\section{BEAM LOADING COMPENSATION IN THE S-BAND ACCELERATORS}

All the S-band linacs will use the same structure design [4]. The parameters of the structure are optimized for the maximum pre-linac beam current since the $8 \mathrm{GeV}$ pre-linacs constitute more than $60 \%$ of total S-band acceleration. The
S-band klystrons are assumed to produce $66 \mathrm{MW}$ of power in a $5 \mu s$ pulse, similar to the SLAC 5045 klystrons. The SLED-I cavity is assumed have an external $Q_{e x t}=2 \times 10^{4}$ with a coupling constant $\beta=5$ yielding a compression factor of approximately four.

\section{1 $\Delta T$ scheme for the $e^{+}$drive and prelinacs}

A power station consists of a pair of klystrons, each feeding a SLED-I system [5]. The SLEDed rf power from the klystron pair is combined and powers six 4-meter accelerator structures. With the pre-linac beam parameters, the optimal fill-time is $625 \mathrm{~ns}$. The SLED-I pulse profile and the energy profile of the beam for the pre-linac compensation are shown in Fig. 2(a) yielding a loaded gradient of $17 \mathrm{MV} / \mathrm{m}$. The best energy deviation using the SLED pulse alone is $4 \times 10^{-3}$. This can be further minimized with a slight amplitude modulation yielding better than $1.3 \times 10^{-3}$.

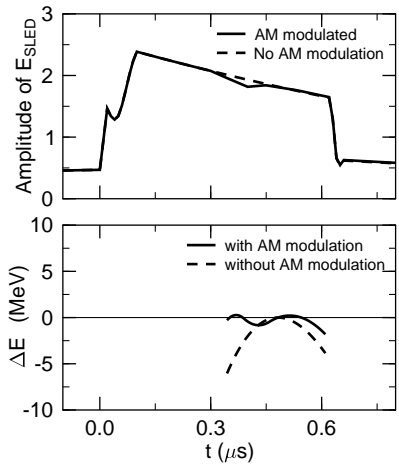

(a)

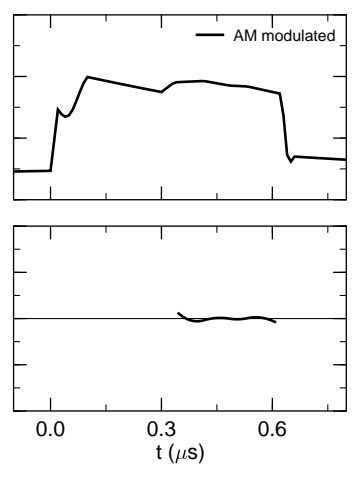

(b)
Figure 2: a) Compensation for the S-band pre-linacs. The SLED-I amplitude can be modulated to further reduce energy spread. b) Compensation for the $e^{-}$booster linac. Amplitude modulation is needed to enhance the slope of the acceleration.

\section{2 $\Delta T$ scheme for the $e^{-}$booster}

The beam current in the $e^{-}$booster linac is $25 \%$ higher than in the pre-linacs. Since the structure is optimized for the pre-linacs, amplitude modulation is necessary to compensate the loading in the booster. Fig. 2(b) shows the SLED-I pulse and the compensated energy deviation in the $e^{-}$booster. The loaded gradient in the $e^{-}$booster is $15.3 \mathrm{MV} / \mathrm{m}$ and the residual energy deviation is about $0.5 \times 10^{-3}$.

\section{BEAM LOADING COMPENSATION IN THE L-BAND ACCELERATORS}

The four L-band linacs will use a 5-meter structure optimized for the $e^{+}$booster linac [4]. The L-band klystrons are assumed to produce a peak power of $75 \mathrm{MW}$ at a pulse length of $5.5 \mu \mathrm{s}$. The SLED-I cavities are simply scaled from the the S-band SLED systems. 


\section{1 $\Delta T$ scheme for the $e^{+}$booster}

An accelerator module for the $e^{+}$booster linac consists of six structures powered by a pair of SLEDed klystrons. The optimal fill-time obtained for $\Delta T$ compensation is $1010 \mathrm{~ns}$ yielding a loaded gradient of $13 \mathrm{MV} / \mathrm{m}$. Without additional amplitude modulation, the minimum energy deviation is $1 \times 10^{-3}$. Figure 3(a) illustrates the energy profiles for the $e^{+}$booster.

\section{2 $\Delta F$ scheme for the $e^{+}$capture}

In the $e^{+}$capture section, the beam loading is too large to be compensated with the $\Delta T$ method alone. Additional compensation is performed using the $\Delta F$ scheme. The capture linac consists of four 5-meter accelerator structures and two $\Delta F$ structures operating at $1428 \pm 1.14 \mathrm{MHz}$. Each $\Delta F$ structure is driven by a single klystron at $50 \mathrm{MW}$ of peak power with SLED-I pulse compression and the loaded gradient in the capture is about $12.7 \mathrm{MV} / \mathrm{m}$. The residual energy deviation is about $1 \times 10^{-2}$ as shown in Fig. 3(b).

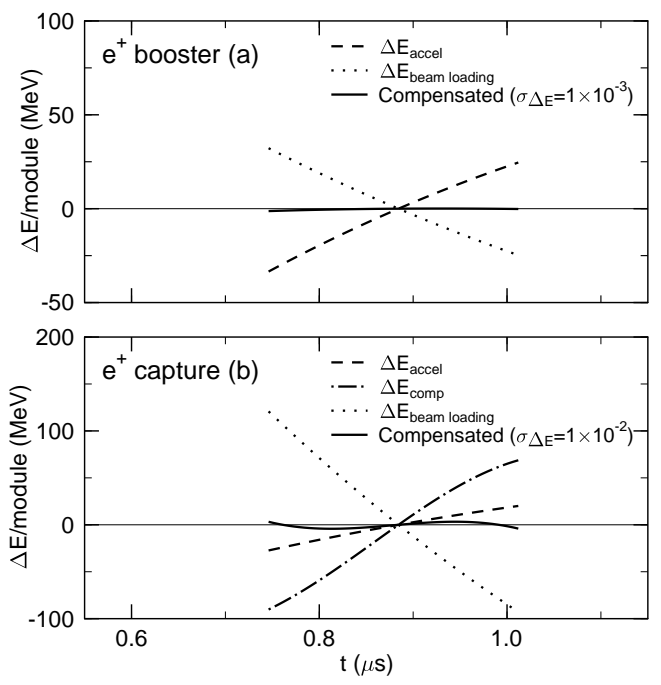

Figure 3: Compensation in the L-band $e^{+}$booster and capture linacs. The fill-time is optimized for the booster linac with $\Delta T$ compensation. Extra beam loading in the capture linac due to higher current is compensated by using the $\Delta F$ scheme. Loaded gradients for both linacs are about $13 \mathrm{MV} / \mathrm{m}$. No amplitude modulation on SLED-I.

\section{3 $\Delta T$ scheme for the compressors}

A module of two (or three) 5-meter structures would be sufficient to generate the voltage ( $100 \mathrm{MV})$ in the compressors. In these systems, both the amplitude and phase are modulated to obtain a uniform compression (amplitude) and loading compensation (phase). The SLED amplitude and phase profiles required for the compressor are shown in Fig. 4 and the energy deviation obtained is $0.11 / E_{0}$, where $E_{0}$ is the energy of the beam in the compressor. The compression voltage is within $1 \%$ deviation. One of the issues with the $\Delta T$ compensation for the compressor linac is the

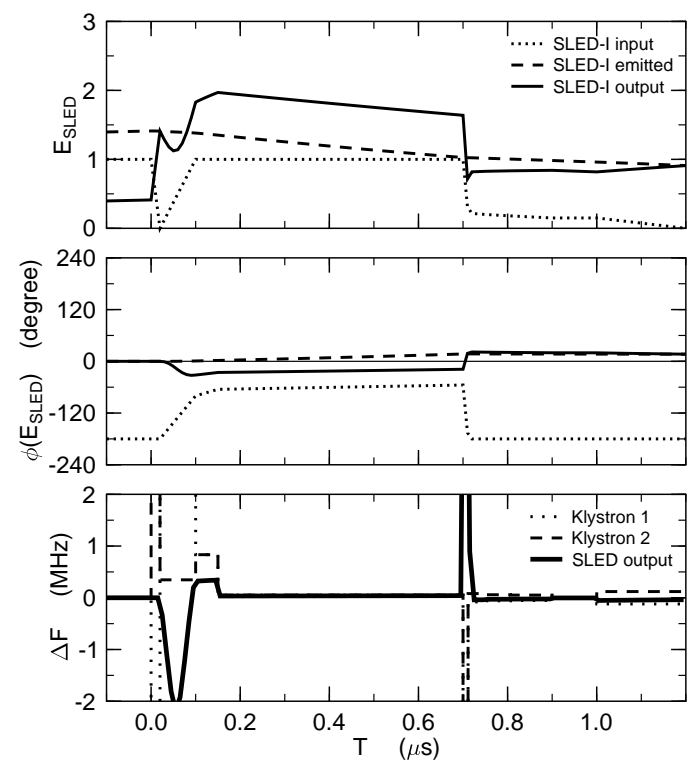

Figure 4: Compensation for the L-band compressor linacs: loaded gradient: $13 \mathrm{MV} / \mathrm{m}$; energy deviation: $0.11 / E_{0}$. Both amplitude and phase are modulated to compensate the beam loading and compression voltages.

frequency offset induced by the phase modulation. Simulation shows that the $\Delta F$ induced by the phase modulation is small in the SLED-I output, thus the effect of this frequency offset is expected to be small.

\section{SUMMARY}

The $\Delta T$ scheme has been shown to be effective in the NLC low rf frequency linacs, except for the $e^{+}$capture linac where $\Delta F$ scheme is required. Once optimized for the nominal beam current, the $\Delta T$ scheme can be used to compensate at different beam loading regimes, such as the beam loading in the $e^{-}$booster, with proper amplitude modulations on the SLED-I pulse. Similarly, the scheme is applicable to these linacs when operating at "zero" beam current. In the future, we need to calculate the tuning tolerances and verify that the dispersive effects are minimal.

\section{REFERENCES}

[1] NLC ZDR Design Group, "Zeroth-Order Design Report for the Next Linear Collider," SLAC Report 474 (1996).

[2] NLC Accelerator Physics Web pages from http://wwwproject.slac.stanford.edu/lc/nlc-tech.html.

[3] Z. D. Farkas and et al, "SLED: A Method of Doubling SLAC's Energy," SLAC-PUB-1453, March 1974.

[4] Z. Li and et al, "Parameter Optimization for the Low RF Linacs of the NLC," this conference.

[5] J. C. Sheppard and et al, "The NLC Injector System," this conference. 